\title{
Impact of Tip Design and Thermocouple Location on the Efficacy and Safety of Radiofrequency Application
}

\author{
Junji Yamaguchi ${ }^{1}$, Masateru Takigawa ${ }^{1}$, Masahiko Goya ${ }^{2}$, Claire Martin ${ }^{3}$, Miki Amemiya ${ }^{1}$, \\ Tasuku Yamamoto ${ }^{1}$, Takuro Nishimura ${ }^{1}$, Rena Nakamura ${ }^{1}$, Yasuhiro Shirai ${ }^{1}$, Susumu Tao ${ }^{1}$, \\ Shinsuke Miyazaki ${ }^{1}$, Yoshihide Takahashi ${ }^{1}$, and Tetsuo Sasano ${ }^{1}$ \\ ${ }^{1}$ Tokyo Medical and Dental University \\ ${ }^{2}$ Tokyo Medical and Dental University Hospital \\ ${ }^{3}$ University of Cambridge
}

October 30, 2021

\begin{abstract}
Background \& Objectives The FlexAbilityTM SE catheter has a laser-cut 8Fr 4-mm flexible tip irrigated through laser-cut kerfs with a thermocouple $0.3 \mathrm{~mm}$ from the distal end. The TactiCathTM SE catheter has an 8Fr 3.5-mm tip and 6-irrigation port with a thermocouple $2.67 \mathrm{~mm}$ proximal to the tip. We investigated the impact of these differences on the efficacy and safety of RF-applications. Methods RF applications at a range of powers (20W, 30W, and 40W), contact forces (5g, $15 \mathrm{~g}$, and 25g), and durations (10-60s) using perpendicular/parallel catheter orientation, were performed in excised porcine hearts. Lesion characteristics and incidence of steam pops were compared. Results 540 lesions were examined. The FlexAbilityTM SE catheter produced smaller lesion depths $(4.0 \mathrm{~mm}$ vs. $4.4 \mathrm{~mm}, \mathrm{p}=0.014$ at $20 \mathrm{~W} ; 4.6 \mathrm{~mm}$ vs. $5.6 \mathrm{~mm}, \mathrm{p}=0.015$ at $30 \mathrm{~W})$, surface areas $(22.7 \mathrm{~mm} 2$ vs. $29.2 \mathrm{~mm} 2$ at $20 \mathrm{~W}, \mathrm{p}=0.005 ; 23.2 \mathrm{~mm} 2$ vs. $28.7 \mathrm{~mm} 2, \mathrm{p}=0.009$ at $30 \mathrm{~W})$ and volumes $(126.1 \mathrm{~mm} 3 \mathrm{vs} .175 .1 \mathrm{~mm} 3$, $\mathrm{p}=0.018$ at $20 \mathrm{~W} ; 183.2 \mathrm{~mm} 3$ vs. $304.3 \mathrm{~mm} 3, \mathrm{p}=0.002$ at $30 \mathrm{~W})$ with perpendicular catheter placement. However, no differences were observed with parallel catheter placement. Steam-pops were significantly less frequently observed with the FlexAbilityTM SE catheter $(4 \%$ vs. $22 \%, \mathrm{p}<0.001)$ irrespective of catheter direction to the tissue. Multivariate analysis showed that use of the TactiCathTM SE catheter, power [?]40W, contact force [?]25g, RF duration $>30$ s, parallel angle and impedance drop [?]20 $\Omega$ were significantly associated with occurrence of steam-pops. Conclusions The FlexAbilityTM SE catheter reduced the risk of steam-pops, but produced smaller lesions with perpendicular catheter placement compared to the TactiCathTM SE catheter.
\end{abstract}

\section{Impact of Tip Design and Thermocouple Location on the Efficacy and Safety of Radiofrequency Application}

Short title: Comparison of Flexibility ${ }^{\mathrm{TM}}$ and TactiCath $^{\mathrm{TM}}$ ablation catheters

Junji Yamaguchi, MD,$^{1^{*}}$ Masateru Takigawa, MD,${ }^{1^{*}}$ Masahiko Goya, MD,${ }^{1}$

Claire Martin, MD² , Miki Amemiya, MD, ${ }^{1}$ Tasuku Yamamoto, MD ${ }^{1}$ Takuro Nichimura, MD,${ }^{1}$ Rena Nakamura, MD, ${ }^{1}$ Yasuhiro Shirai, MD, ${ }^{1}$ Susumu Tao, MD,${ }^{1}$

Shinsuke Miyazaki, MD, ${ }^{1}$ Yoshihide Takahashi, MD,${ }^{1}$

Tetsuo Sasano, $\mathrm{MD}^{1}$

${ }^{1}$ Department of Cardiovascular Medicine, Tokyo Medical and Dental University Hospital, Tokyo, Japan.

${ }^{2}$ Royal Papworth Hospital, Cambridge, UK

${ }^{*}$ These authors contributed equally to this work. 


\section{Conflicts of interest}

Drs. Takigawa and Takahashi received endowments from Medtronic Japan, Boston Scientific, Japan Lifeline, and WIN international. No other authors have conflict of interest to declare.

\section{Funding}

This work was partially supported by JSPS KAKENHI Grant Number JP20K17074.

Corresponding Author: Masateru Takigawa, MD, $\mathrm{PhD}$.

Department of Cardiovascular Medicine, Tokyo Medical and Dental University Hospital, 1-5-45 Yushima, Bunkyo-ku, Tokyo 113-8510, Japan.

TEL: +(81)3-3813-6111 FAX: +(81)3-5803-0110 E-mail: teru.takigawa@gmail.com

\section{Abstract \\ Background \& Objectives}

The FlexAbility ${ }^{\mathrm{TM}} \mathrm{SE}$ catheter has a laser-cut $8 \mathrm{Fr}$ 4-mm flexible tip irrigated through laser-cut kerfs with a thermocouple $0.3 \mathrm{~mm}$ from the distal end. The TactiCath ${ }^{\mathrm{TM}} \mathrm{SE}$ catheter has an 8Fr 3.5-mm tip and 6 -irrigation port with a thermocouple $2.67 \mathrm{~mm}$ proximal to the tip. We investigated the impact of these differences on the efficacy and safety of RF-applications.

\section{Methods}

$\mathrm{RF}$ applications at a range of powers $(20 \mathrm{~W}, 30 \mathrm{~W}$, and $40 \mathrm{~W})$, contact forces $(5 \mathrm{~g}, 15 \mathrm{~g}$, and $25 \mathrm{~g})$, and durations (10-60s) using perpendicular/parallel catheter orientation, were performed in excised porcine hearts. Lesion characteristics and incidence of steam pops were compared.

\section{Results}

540 lesions were examined. The FlexAbility ${ }^{\mathrm{TM}}$ SE catheter produced smaller lesion depths (4.0mm vs. $4.4 \mathrm{~mm}, \mathrm{p}=0.014$ at $20 \mathrm{~W} ; 4.6 \mathrm{~mm}$ vs. $5.6 \mathrm{~mm}, \mathrm{p}=0.015$ at $30 \mathrm{~W})$, surface areas $\left(22.7 \mathrm{~mm}^{2}\right.$ vs. $29.2 \mathrm{~mm}^{2}$ at $20 \mathrm{~W}, \mathrm{p}=0.005 ; 23.2 \mathrm{~mm}^{2}$ vs. $28.7 \mathrm{~mm}^{2}, \mathrm{p}=0.009$ at $\left.30 \mathrm{~W}\right)$ and volumes $\left(126.1 \mathrm{~mm}^{3}\right.$ vs. $175.1 \mathrm{~mm}^{3}, \mathrm{p}=0.018$ at $20 \mathrm{~W} ; 183.2 \mathrm{~mm}^{3}$ vs. $304.3 \mathrm{~mm}^{3}, \mathrm{p}=0.002$ at $30 \mathrm{~W}$ ) with perpendicular catheter placement. However, no differences were observed with parallel catheter placement. Steam-pops were significantly less frequently observed with the FlexAbility ${ }^{\mathrm{TM}} \mathrm{SE}$ catheter $(4 \%$ vs. $22 \%, \mathrm{p}<0.001)$ irrespective of catheter direction to the tissue. Multivariate analysis showed that use of the TactiCath ${ }^{\mathrm{TM}}$ SE catheter, power [?] $40 \mathrm{~W}$, contact force [?]25g, RF duration $>30$ s, parallel angle and impedance drop [?]20 $\Omega$ were significantly associated with occurrence of steam-pops.

\section{Conclusions}

The FlexAbility ${ }^{\mathrm{TM}} \mathrm{SE}$ catheter reduced the risk of steam-pops, but produced smaller lesions with perpendicular catheter placement compared to the TactiCath ${ }^{\mathrm{TM}} \mathrm{SE}$ catheter.

\section{Keywords}

Catheter ablation; FlexAbility ${ }^{\mathrm{TM}}$ SE; TactiCath $^{\mathrm{TM}}$ SE; Lesion size

\section{Introduction}

Radiofrequency (RF) catheter ablation is an established treatment for cardiac arrhythmias. Optimal lesion formation and avoidance of excessive heating are necessary for effective ablation (1). Multiple factors including power, duration, catheter-tissue contact force $(\mathrm{CF})$, electrode diameter, ablation circuit impedance, irrigation flow, tissue thickness, and myocardial blood flow have been reported to be associated with lesion characteristics $(2,3)$. 
Recently, a variety of new catheters have been developed and used in a clinical setting. Since each catheter has unique features, ablation lesion characteristics and complication rates may differ among catheters. Operators must understand the features of each catheter to optimize efficacy and safety. The open-irrigated ablation catheters, FlexAbility ${ }^{\mathrm{TM}} \mathrm{SE}$ and TactiCath ${ }^{\mathrm{TM}} \mathrm{SE}$ (Abbott, St. Paul, MN), have different irrigation flows, electrode sizes and locations of the thermocouple, but little is known about the effect of these differences. The aim of this study was to investigate the lesion characteristics and adverse events of these catheters in ex vivo conditions.

\section{Methods}

\section{Irrigated Ablation Catheters}

Two open-irrigated ablation catheters were compared. Images of the catheter tips are shown in Figure 1A. The FlexAbility ${ }^{\mathrm{TM}} \mathrm{SE}$ ablation catheter has a laser-cut 4-mm flexible $8 \mathrm{Fr}$ tip, which is irrigated from the proximal to distal end through laser-cut kerfs, and from four holes on the distal end of the tip. The TactiCath $^{\text {TM }}$ SE ablation catheter has a 3.5-mm 8Fr tip and has 6-hole irrigation holes on the side of the tip. Both catheters have a single thermocouple embedded within the tip for temperature monitoring, and the distance from the tip is $0.3 \mathrm{~mm}$ in the FlexAbility ${ }^{\mathrm{TM}} \mathrm{SE}$ catheter and $2.64 \mathrm{~mm}$ in the TactiCath ${ }^{\mathrm{TM}} \mathrm{SE}$ catheter.

\section{Experimental Model}

A circulating saline bath $(400 \mathrm{~mL} / \mathrm{min}, \mathrm{NaCl} 3.5 \mathrm{~g} / \mathrm{L}$ ) isotonic to blood, a submersible load cell (KW-201, Tanita, Tokyo, Japan), and a deflectable sheath were assembled. The load cell was installed on the bottom of the bath with $5.0 \mathrm{~L}$ saline at $37^{\circ} \mathrm{C}$, and a section of the left ventricular myocardium was placed on the ground plate on a load cell apparatus. CF applied to the overlying myocardial tissue through the ablation catheter was measured by the load cell in order to provide the same conditions between the TactiCath ${ }^{\mathrm{TM}}$ $\mathrm{SE}$ (which has direct CF-sensing capability) and FlexAbility ${ }^{\mathrm{TM}} \mathrm{SE}$ catheters. The accuracy of CF in this model was verified by a CF-sensing catheter with EnSite Precision (Abbott) mapping system as shown in Supplementary Figure 1. Briefly, the correlation coefficient between the CF measured by the load cell and $\mathrm{CF}$ in the TactiCath ${ }^{\mathrm{TM}} \mathrm{SE}$ catheter was $\mathrm{R}=0.97$ for perpendicular catheter placement and $\mathrm{R}=0.77$ for parallel catheter placement. Temperature, power and impedance values were continuously monitored during RF delivery using the EnSite Precision cardiac mapping system.

\section{RF applications}

A calibrated roller pump (CoolPoint, Abbott) connected to the catheter delivered saline solution at 20 $\mathrm{ml} / \mathrm{min}$ for the FlexAbility ${ }^{\mathrm{TM}} \mathrm{SE}^{\mathrm{M}}$ catheter and $17-30 \mathrm{ml} / \mathrm{min}$ for the TactiCath ${ }^{\mathrm{TM}} \mathrm{SE}$ catheter $(17 \mathrm{ml} / \mathrm{min}$ at $20 \mathrm{~W}$ and $30 \mathrm{~W}, 30 \mathrm{ml} / \mathrm{min}$ at $40 \mathrm{~W}$ ) during RF delivery as recommended by the manufacturer for clinical use of each catheter. An Ampere RF generator (Abbott) was connected to deliver $550 \mathrm{kHz}$ unmodulated sine-wave RF energy pulses in a temperature-controlled mode (maximum temperature 40). Different power settings, contact intensities, RF duration, and catheter orientation were used during the experiment, as discussed in the following sections.

Ablation protocol

To obtain a range of lesion sizes, myocardial lesions were created at separate sites with contact force set at 5, 15 and $25 \mathrm{~g}$, and power settings set at 20,30 and $40 \mathrm{~W}$. For each contact force and power setting, RF delivery was delivered for 10,20,30, 45 and 60 seconds. The catheter was placed perpendicularly and parallelly to the tissue (Figure 1B). Three lesions for each combination of settings were created (270 lesions for each catheter). After creating every lesion, the ablation electrode and the electrode-tissue interface were examined for char formation. The incidence of an audible pop was noted.

Lesion assessment

After RF delivery, lesion surface was measured as shown in Figure 1C. The myocardium was cross-sectioned along the surface length at the level of each lesion. The cross-sectioned area was also measured as shown in 
Figure 1C. Each lesion was measured with a dial caliper with a resolution of $0.1 \mathrm{~mm}$ by one observer; the observer was not blinded to the lesion protocol. Surface area and lesion volume were calculated from the following formulae:

Lesion volume $=(1 / 6) \mathrm{x} \pi \times\left(\mathrm{e}^{2} \times \mathrm{d}+\mathrm{c} \times \mathrm{a}^{2} / 2\right)$

Lesion surface area $=\pi \times \mathrm{a} / 2 \times \mathrm{b} / 2(4)$

Lesions with steam-pops were excluded from the lesion size analysis.

Statistical analysis

The data are presented as median (interquartile range). Continuous variables were compared by Wilcoxon rank-sum test. Categorical variables were compared by an $\chi^{2}$ test or Fisher's exact test. Significant differences were further evaluated by using Bonferroni's method for pairwise multiple comparisons. A P-value $<0.05$ was considered statistically significant. All statistical analyses were performed with $\mathrm{R}$ software (The R Foundation for Statistical Computing, Vienna, Austria).

\section{Results}

\section{RF delivery}

A summary of the total lesion count ( $\mathrm{N}=270$ lesions for each catheter) including steam-pops and char formation and parameters of RF-delivery is shown in Table 1. Average power was significantly higher with the TactiCath ${ }^{\mathrm{TM}} \mathrm{SE}$ catheter compared to FlexAbility ${ }^{\mathrm{TM}} \mathrm{SE}$ (19W vs. $20 \mathrm{~W}, \mathrm{p}=0.004$ at $20 \mathrm{~W}$ setting; $27 \mathrm{~W}$ vs. $29 \mathrm{~W}, \mathrm{p}<0.001$ at $30 \mathrm{~W}$ setting; $34.5 \mathrm{~W}$ vs. $38 \mathrm{~W}, \mathrm{p}<0.001$ at $40 \mathrm{~W}$ setting), reflecting the lower temperature control rate $(0 \%$ vs. $0 \%$ at $20 \mathrm{~W} ; 1.1 \%$ vs. $20 \%$, p $<0.001$ at $30 \mathrm{~W} ; 3.3 \%$ vs. $46.7 \%$ at $40 \mathrm{~W})$. Steam-pops were observed more frequently with the TactiCath ${ }^{\mathrm{TM}} \mathrm{SE}$ catheter at $30 \mathrm{~W}\left(1.1 \%\right.$ in FlexAbility ${ }^{\mathrm{TM}} \mathrm{SE}$ vs. $8.9 \%$ in TactiCath $^{\mathrm{TM}}$ SE, $\left.\mathrm{p}=0.034\right)$ and 40W (13.3\% in FlexAbility ${ }^{\mathrm{TM}} \mathrm{SE}$ vs. $57.8 \%$ in TactiCath $\left.^{\mathrm{TM}} \mathrm{SE}, \mathrm{p}<0.001\right)$.

\section{Lesion characteristics}

Lesion characteristics are summarized in Table 2. Lesions with steam-pops were excluded from the lesion metrics. At 20W, the TactiCath ${ }^{\mathrm{TM}} \mathrm{SE}$ catheter provided larger lesions than the FlexAbility ${ }^{\mathrm{TM}} \mathrm{SE}$ catheter (depth: $4.0 \mathrm{~mm}$ vs. $3.8 \mathrm{~mm}, \mathrm{p}=0.021$, surface area: $26.4 \mathrm{~mm}^{2}$ vs. $30.0 \mathrm{~mm}^{2}, \mathrm{p}=0.068$; lesion volume: $175.1 \mathrm{~mm}^{3}$ vs. $\left.149.0 \mathrm{~mm}^{3}, \mathrm{p}=0.067\right)$. When the catheter was placed perpendicularly to the tissue, lesion depth $(4.4 \mathrm{~mm}$ vs. $4.0 \mathrm{~mm}, \mathrm{p}=0.014)$, surface area $\left(29.2 \mathrm{~mm}^{2}\right.$ vs. $\left.22.7 \mathrm{~mm}^{2}, \mathrm{p}=0.005\right)$ and lesion volume $\left(175.1 \mathrm{~mm}^{3}\right.$ vs. $126.1 \mathrm{~mm}^{3}, \mathrm{p}=0.018$ ) were significantly larger with the TactiCath ${ }^{\mathrm{TM}} \mathrm{SE}$ catheter. However, when the catheter was placed parallelly, no difference in lesion characteristics was observed between these two catheters. The same findings were also observed at $30 \mathrm{~W}$ and $40 \mathrm{~W}$ settings. However, comparison of lesion sizes in $40 \mathrm{~W}$ was less reliable due to exclusion of multiple points due to more frequent steam-pops with the TactiCath ${ }^{\mathrm{TM}} \mathrm{SE}$ catheter $(52 / 90[57.8 \%]$ vs. $11 / 90[12.2 \%], \mathrm{p}<0.001)$.

A positive relationship between lesion volume and RF duration was observed for both catheters as shown in Figure 2. With perpendicular catheter placement, lesions were generally larger with the TactiCath ${ }^{\mathrm{TM}} \mathrm{SE}$ than FlexAbility ${ }^{\mathrm{TM}}$ SE catheters (Figure 2A,C), while no significant difference in lesion size was observed with parallel catheter placement (Figure 2B,D).

\section{Temperature control}

As shown in Table 1, RF-regulation due to the temperature-control setting was more frequently observed with the FlexAbility ${ }^{\mathrm{TM}} \mathrm{SE}$ catheter at $30 \mathrm{~W}(20 \%$ vs. $0 \%, \mathrm{p}<0.001)$ and $40 \mathrm{~W}(46.7 \%$ vs. $3.3 \%, \mathrm{p}<0.001)$ settings. Further, as shown in Table 3, this finding was more marked with perpendicular catheter placement than with a parallel angle at $30 \mathrm{~W}(31.1 \%$ vs. $8.9 \%, \mathrm{p}=0.016)$ and $40 \mathrm{~W}(66.7 \%$ vs. $26.7 \%)$ settings, which resulted in lower average power delivery with perpendicular catheter placement. In contrast, RF-delivery was not frequently regulated by the temperature-control setting regardless of the catheter orientation in any power settings with the TactiCath ${ }^{\mathrm{TM}} \mathrm{SE}$ catheter (Table 3). 


\section{Adverse event rates}

As shown in Table 1, no char formation was observed but 72/540 (13.3\%) (n=12 with FlexAbility ${ }^{\text {TM }}$ SE and $\mathrm{n}=60$ with TactiCath $\left.{ }^{\mathrm{TM}} \mathrm{SE}, \mathrm{P}<0.001\right)$ steam-pops were observed. At both $30 \mathrm{~W}(1.1 \%$ vs. 8.9\%, $\mathrm{p}=0.034)$ and $40 \mathrm{~W}(12.2 \%$ vs. $57.8 \%, \mathrm{p}<0.001)$ settings, use of the FlexAbility ${ }^{\mathrm{TM}} \mathrm{SE}$ catheter significantly reduced the incidence of steam-pops.

As shown in Table 4, power [?]40W (Odds ratio (OR) 53.0, p<0.001), contact force[?]25g (OR 2.1, p=0.047), $\mathrm{RF}$ duration $>30 \mathrm{~s}$ (OR 8.0, p<0.001), parallel angle (OR 2.1, p=0.045), and impedance drop [?]20 $(\mathrm{OR}$ $3.0, \mathrm{p}=0.005)$ remained as positive predictors of steam-pop but use of the FlexAbility ${ }^{\mathrm{TM}} \mathrm{SE}$ catheter $(\mathrm{OR}$ $0.07, \mathrm{p}<0.001$ ) was negatively associated with steam-pops by multivariate analysis.

\section{Discussion}

To the best of our knowledge, this is the first study comparing the lesion metrics and safety profiles of two open irrigated ablation catheters from Abbott Medical (FlexAbility ${ }^{\mathrm{TM}} \mathrm{SE}_{\mathrm{S}}$ an TactiCath $^{\mathrm{TM}} \mathrm{SE}$ ), demonstrating that:

Compared to the TactiCath ${ }^{\mathrm{TM}}$ SE catheter, use of the FlexAbility ${ }^{\mathrm{TM}}$ SE catheter leads to a significantly lower incidence of steam-pops, but produces smaller lesions with perpendicular catheter placement.

Regulation of RF-delivery due to the temperature-control setting was more frequently observed with the FlexAbility $^{\mathrm{TM}}$ SE catheter.

Difference in lesion characteristics between catheters

In our study, the FlexAbility ${ }^{\mathrm{TM}} \mathrm{SE}$ catheter produced smaller lesions than the TactiCath ${ }^{\mathrm{TM}} \mathrm{SE}^{\mathrm{cath}} \mathrm{T}_{\mathrm{te}}$, especially for perpendicular catheter placement (Figure 3A). A higher rate of temperature control in the FlexAbility ${ }^{\mathrm{TM}} \mathrm{SE}$ catheter, resulting in a lower average power, can partially explain this finding. However, lesion size was smaller with the FlexAbility ${ }^{\mathrm{TM}} \mathrm{SE}$ catheter with perpendicular catheter placement even at a $20 \mathrm{~W}$ setting, in which temperature control was not observed. Another possible explanation is that the FlexAbility ${ }^{\mathrm{TM}} \mathrm{SE}$ catheter has a larger tip size of $8 \mathrm{Fr} 4 \mathrm{~mm}$, compared to $8 \mathrm{Fr} 3.5 \mathrm{~mm}$ for the TactiCath ${ }^{\mathrm{TM}}$ $\mathrm{SE}$ catheter. In general, the current density of a larger tip catheter is lower for the same power setting, and therefore the current density of the FlexAbility ${ }^{\mathrm{TM}} \mathrm{SE}$ catheter should be lower, resulting in smaller lesion creation under the same power settings (5). Additionally, Flexability ${ }^{\mathrm{TM}}$ SE has more efficient cooling effect than TactiCath ${ }^{\mathrm{TM}} \mathrm{SE}$ due to differences in irrigation profile.

Frequency of steam-pops

Our analysis suggests that high power, high contact force, long RF duration and large impedance decrease were independent predictors of steam-pop, which is consistent with previous studies (6-9). Additionally, our study demonstrated that parallel catheter placement was positively associated and use of the FlexAbility ${ }^{\mathrm{TM}} \mathrm{SE}$ catheter was negatively associated with steam-pops. The safety of the TactiCath ${ }^{\mathrm{TM}} \mathrm{SE}$ catheter has been shown in a randomized controlled clinical study for paroxysmal atrial fibrillation patients (10). However, the superiority of the FlexAbility ${ }^{\mathrm{TM}} \mathrm{SE}$ catheter in avoiding steam-pops was demonstrated in the present study (20W:0\% 30W:1.1\% 40W: 12.2\% in FlexAbility ${ }^{\mathrm{TM}} \mathrm{SE}$, and 20W:0\% 30W:8.9\% 40W:57.8\% in TactiCath ${ }^{\mathrm{TM}} \mathrm{SE}$ ), whilst the incidence of steam-pops with the TactiCath ${ }^{\mathrm{TM}} \mathrm{SE}$ catheter was similar to the previous reports of TactiCath ${ }^{\mathrm{TM}}$ Quartz catheter (Abbott, St. Paul, MN) and IntellaNav MiFi OI catheter (Boston Scientific, Marlborough, MA) (11,12).

Since temperature control plays an essential role in preventing steam-pops and char formation, the FlexAbility ${ }^{\mathrm{TM}}$ SE catheter benefits from a high temperature control rate. Although parallel catheter placement was an independent predictor of steam-pop in our study, this finding has not yet been confirmed by other studies. One study reported that greater tissue heating with a perpendicular compared to parallel catheter placement is accompanied by increased incidence of steam pops (13). In contrast, larger lesion volume and larger impedance decrease, known as predictors of steam pop, have been observed more frequently with a parallel than perpendicular catheter placement in several studies $(14,15)$. 
Although the ultimate reason for the higher incidence of steam-pops with a parallel catheter placement in our study is unknown, we propose the following possibilities. With the TactiCath ${ }^{\mathrm{TM}} \mathrm{SE}$ catheter, the current density is known to be higher at the proximal region of the tip than the distal region. When the catheter is placed parallel to the tissue, the area of high current density connects with the tissue. In the TactiCath ${ }^{\mathrm{TM}} \mathrm{SE}$ catheter, a lower incidence of RF regulation based on the temperature-control setting may result in a higher average power with parallel catheter placement, leading to increased incidence of steam pops. However, the incidence of steam-pops with the FlexAbility ${ }^{\mathrm{TM}} \mathrm{SE}$ catheter was generally low, and further studies including tissue temperature monitoring are required to better understand the impact of catheter contact angle.

Regulation of RF-delivery under the temperature-control setting

As described above, regulation of RF-delivery under the temperature-control setting may impact both lesion characteristics and steam-pop events. We demonstrate in this study that this regulation was more frequently observed with the FlexAbility $^{\mathrm{TM}}$ SE catheter than the TactiCath ${ }^{\mathrm{TM}}$ SE catheter $(60$ [22\%] vs. 4 [4\%], $\mathrm{p}<0.001$ ). Firstly, this may be due to the location of the thermocouple in determining the sensitivity to thermal change in the tissue during RF delivery. The location of the thermocouple is only $0.3 \mathrm{~mm}$ proximal to the tip in the FlexAbility ${ }^{\mathrm{TM}} \mathrm{SE}$ catheter, compared to $2.64 \mathrm{~mm}$ in the TactiCath ${ }^{\mathrm{TM}} \mathrm{SE}$ catheter. Our results compliment a previous study showing that the tip temperature of the FlexAbility ${ }^{\mathrm{TM}} \mathrm{SE}$ catheter provides better safety feedback compared to the Thermocool ${ }^{\mathrm{TM}}$ and ThermoCool SF ${ }^{\mathrm{TM}}$ catheters, where the thermocouple is located $2.0 \mathrm{~mm}$ and $6.6 \mathrm{~mm}$ from the distal end of the tip (16).

Termination of RF-delivery in the FlexAbility ${ }^{\mathrm{TM}}$ SE catheter was more frequently observed with perpendicular catheter placement. This may be due to the localized temperature measurement capability of the catheter due to the distal placement and proximity to tissue of the thermocouple. When the catheter is placed parallel to the tissue, the kerfs direct irrigation flow towards the tissue surface thus enabling effective cooling of the tissue (16). However, when the catheter is placed perpendicularly, the irrigation flow spreads from proximal to distal, which may lead to relatively insufficient cooling (Figure 4, see supplemental VIDEO).

\section{Clinical implications}

The present study suggests that RF application should be performed in accordance with the characteristics of each catheter (Figure 3B). The FlexAblility ${ }^{\mathrm{TM}} \mathrm{SE}$ catheter has a thermocouple at the distal end of the tip, which enables it to monitor tissue temperature more accurately, therefore safer RF application can be performed. Although lesion volume from the FlexAbility ${ }^{\mathrm{TM}} \mathrm{SE}$ catheter is similar to that of the TactiCath ${ }^{\mathrm{TM}}$ SE catheter with parallel catheter placement, it becomes smaller when the catheter is placed perpendicularly. The TactiCath ${ }^{\mathrm{TM}} \mathrm{SE}$ catheter has an advantage in creating larger lesions even with a perpendicular placement, but in order to ensure safety, risk factors for steam-pops such as impedance decrease and contact force should be carefully monitored, because of the lower sensitivity in measuring tissue temperature due to the proximal location of the thermocouple.

\section{Limitations}

The primary limitation is that this study was performed on an in vitro model and the results cannot be translated directly into clinical practice. However, these limitations similarly affect both catheters, so that differences between the two catheters may be preserved.

Secondly, CF was measured using a submersible load cell and not using a catheter incorporating a CF sensor in both catheters because of the absence of contact force sensing technology in the FlexAbility ${ }^{\mathrm{TM}} \mathrm{SE}$ catheter. However, this technique did provide a reliable correlation to CF for the TactiCath ${ }^{\mathrm{TM}} \mathrm{SE}$ catheter (for perpendicular angle $\mathrm{R}=0.97$ and for parallel angle $\mathrm{R}=0.77$ ).

Finally, to avoid frequent temperature-control in ex-vivo setting, the flow rate $(=20 \mathrm{ml} / \mathrm{min})$ was used for FlexAbility ${ }^{\mathrm{TM}} \mathrm{SE}$ in this study, which may result in smaller lesions.

\section{Conclusions}


Although the TactiCath ${ }^{\mathrm{TM}}$ SE catheter produces larger lesions, the FlexAbility ${ }^{\mathrm{TM}}$ SE catheter may reduce adverse events during RF-delivery. Catheter angle to the tissue should be taken into consideration with the FlexAbility ${ }^{\mathrm{TM}} \mathrm{SE}$ catheter in order to maintain a lesion volume similar to the TactiCath $^{\mathrm{TM}} \mathrm{SE}$ catheter.

\section{Acknowlegement}

We appreciate a technical support for this experiment from Mr. Kazuyoshi Moriya, Mr. Masashi Uemoto, Mr. Ryoto Shiga, Miss. Miso Suzuki, and Miss. Lisa Sakurai from Abbott Medical Japan LIS.

\section{References}

1. El Haddad M, Taghji P, Phlips T, et al. Determinants of acute and late pulmonary vein reconnection in contact force-guided pulmonary vein isolation: identifying the weakest link in the ablation chain. Circ Arrhythm Electrophysiol. 2017;10:e004867.

2. Ariyarathna N, Kumar S, Thomas SP, Stevenson WG, Michaud GF. Role of Contact Force Sensing in Catheter Ablation of Cardiac Arrhythmias: Evolution or History Repeating Itself? JACC Clin Electrophysiol 2018; 4: 707-723.

3. Petersen HH, Chen X, Pietersen A, Svendsen JH, Haunsø S. Tissue temperatures and lesion size during irrigated tip catheter radiofrequency ablation: an in vitro comparison of temperature-controlled irrigated tip ablation, power-controlled irrigated tip ablation, and standard temperature-controlled ablation. Pacing Clin Electrophysiol 2000; 23: 8-17.

4. Nakagawa H, Yamanashi WS, Pitha JV, Arruda M, Wang X, Ohtomo K, Beckman KJ, McClelland JH, Lazzara R, Jackman WM. Comparison of in vivo tissue temperature profile and lesion geometry for radiofrequency ablation with a saline-irrigated electrode versus temperature control in a canine thigh muscle preparation. Circulation. 1995; 91:2264-2273.

5. Barkagan M, Leshem E, Rottmann M, Sroubek J, Shapira-Daniels A, Anter E. Expandable Lattice Electrode Ablation Catheter: A Novel Radiofrequency Platform Allowing High Current at Low Density for Rapid, Titratable, and Durable Lesions. Circ Arrhythm Electrophysiol 2019; 12: e007090.

6. Ikeda A, Nakagawa H, Lambert H, Shah DC, Fonck E, Yulzari A, Sharma T, Pitha JV, Lazzara R, Jackman WM. Relationship between catheter contact force and radiofrequency lesion size and incidence of steam pop in the beating canine heart: electrogram amplitude, impedance, and electrode temperature are poor predictors of electrode-tissue contact force and lesion size. Circ Arrhythm Electrophysiol 2014; 7: 1174-80.

7. Seiler J, Roberts-Thomson KC, Raymond JM, Vest J, Delacretaz E, Stevenson WG. Steam pops during irrigated radiofrequency ablation: feasibility of impedance monitoring for prevention. Heart Rhythm 2008; 5: 1411-6.

8. Theis C, Rostock T, Mollnau H, Sonnenschein S, Himmrich E, Kämpfner D, Ocete BQ, Bock K, Münzel T, Konrad T. The Incidence of Audible Steam Pops Is Increased and Unpredictable With the ThermoCool@ Surround Flow Catheter During Left Atrial Catheter Ablation: A Prospective Observational Study. J Cardiovasc Electrophysiol 2015; 26: 956-962.

9. Bourier F, Popa M, Kottmaier M, Maurer S, Bahlke F, Telishevska M, Lengauer S, Koch-Büttner K, Kornmayer M, Risse E, Brkic A, Reents T, Hessling G, Deisenhofer I. RF electrode-tissue coverage significantly influences steam pop incidence and lesion size. J Cardiovasc Electrophysiol 2021; 32:1594-1599.

10. Reddy VY, Dukkipati SR, Neuzil P, Natale A, Albenque JP, Kautzner J, Shah D, Michaud G, Wharton M, Harari D, Mahapatra S, Lambert H, Mansour M. Randomized, Controlled Trial of the Safety and Effectiveness of a Contact Force-Sensing Irrigated Catheter for Ablation of Paroxysmal Atrial Fibrillation: Results of the TactiCath Contact Force Ablation Catheter Study for Atrial Fibrillation (TOCCASTAR) Study. Circulation 2015; 132: 907-15. 
11. Calzolari V, De Mattia L, Indiani S, Crosato M, Furlanetto A, Licciardello C, Squasi PAM, Olivari Z. In Vitro Validation of the Lesion Size Index to Predict Lesion Width and Depth After Irrigated Radiofrequency Ablation in a Porcine Model. JACC Clin Electrophysiol 2017; 3: 1126-1135.

12. Tsutsui K, Kawano D, Mori H, Kato R, Ikeda Y, Sumitomo N, Fukaya H, Iwanaga S, Nakano S, Muramatsu T, Matsumoto K. Characteristics and optimal ablation settings of a novel, contact-force sensing and local impedance-enabled catheter in an ex vivo perfused swine ventricle model. J Cardiovasc Electrophysiol 2021. doi: 10.1111/jce.15253. Epub ahead of print. PMID: 34559441.

13. Ching KC, Elayi CS, Oussama MW, Khan MN, Conor B, Karen P, Callahan T, Zakaib J, Fahmy TS, Schweikert RA, Saliba W, Dresing T, Burkhardt JD, Cummings JE, Arruda M, Natale A: Steam pops in real life: Confessions of open irrigated catheters. Heart Rhythm 2007;4:S11(abstract).

14. Knecht S, Reichlin T, Pavlovic N, Schaer B, Osswald S, Sticherling C, Kühne M. Contact force and impedance decrease during ablation depends on catheter location and orientation: insights from pulmonary vein isolation using a contact force-sensing catheter. J Interv Card Electrophysiol 2015;43:297-306.

15. González-Suárez A, Berjano E, Guerra JM, Gerardo-Giorda L. Computational Modeling of OpenIrrigated Electrodes for Radiofrequency Cardiac Ablation Including Blood Motion-Saline Flow Interaction. PLoS One 2016; 11:e0150356.

16. Winterfield JR, Jensen J, Gilbert T, Marchlinski F, Natale A, Packer D, Reddy V, Mahapatra S, Wilber DJ. Lesion Size and Safety Comparison Between the Novel Flex Tip on the FlexAbility Ablation Catheter and the Solid Tips on the ThermoCool and ThermoCool SF Ablation Catheters. J Cardiovasc Electrophysiol 2016; $27: 102-9$.

\section{Tables}

Table 1. Summary

\begin{tabular}{|c|c|c|c|}
\hline & Number of lesions & Number of lesions & \\
\hline & FlexAbility $^{\mathrm{TM}} \mathrm{SE}$ & TactiCath $^{\mathrm{TM}} \mathrm{SE}$ & P-value \\
\hline $20 \mathrm{~W}$ & 90 & 90 & \\
\hline $30 \mathrm{~W}$ & 90 & 90 & \\
\hline $40 \mathrm{~W}$ & 90 & 90 & \\
\hline \multirow[t]{3}{*}{ Total } & 270 & 270 & \\
\hline & Average power (W) & Average power (W) & \\
\hline & FlexAbility $^{\mathrm{TM}} \mathrm{SE}$ & TactiCath $^{\mathrm{TM}} \mathrm{SE}$ & $\mathrm{P}$-value \\
\hline $20 \mathrm{~W}$ & $19[19-20]$ & $20[19-20]$ & 0.004 \\
\hline $30 \mathrm{~W}$ & $27[25-28]$ & 29 [29-30] & $<0.001$ \\
\hline \multirow[t]{3}{*}{$40 \mathrm{~W}$} & $34.5[31-37]$ & $38[38-39]$ & $<0.001$ \\
\hline & Temperature control & Temperature control & \\
\hline & FlexAbility $^{\mathrm{TM}} \mathrm{SE}$ & TactiCath $^{\mathrm{TM}} \mathrm{SE}$ & P-value \\
\hline $20 \mathrm{~W}$ & 0 & 0 & 1 \\
\hline $30 \mathrm{~W}$ & $18(20.0 \%)$ & 0 & $<0.001$ \\
\hline \multirow[t]{3}{*}{$40 \mathrm{~W}$} & $42(46.7 \%)$ & $3(3.3 \%)$ & $<0.001$ \\
\hline & Steam-pop & Steam-pop & \\
\hline & FlexAbility ${ }^{\mathrm{TM}} \mathrm{SE}$ & TactiCath $^{\mathrm{TM}} \mathrm{SE}$ & $\mathrm{P}$-value \\
\hline $20 \mathrm{~W}$ & 0 & 0 & 1 \\
\hline $30 \mathrm{~W}$ & $1(1.1 \%)$ & $8(8.9 \%)$ & 0.034 \\
\hline 40W & $11(12.2 \%)$ & $52(57.8 \%)$ & $<0.001$ \\
\hline Char formation & 0 & 0 & 1 \\
\hline
\end{tabular}

Table 2. Lesion size 


\begin{tabular}{|c|c|c|c|}
\hline & FlexAbility $^{\mathrm{TM}} \mathrm{SE}$ & TactiCath $^{\mathrm{TM}} \mathrm{SE}$ & $\mathrm{P}$-value \\
\hline \multicolumn{4}{|l|}{$20 \mathrm{~W}$} \\
\hline Total & $\mathrm{N}=90$ & $\mathrm{~N}=90$ & \\
\hline Lesion depth & $3.8[2.8-4.4]$ & $4.0[3.1-5.0]$ & 0.021 \\
\hline Surface area & $26.4[20.3-34.7]$ & 30.0 [23.9-34.5] & 0.068 \\
\hline Lesion volume & $149.0[90.3-212.1]$ & $175.1[99.7-279.5]$ & 0.067 \\
\hline Perpendicular & $\mathrm{N}=45$ & $\mathrm{~N}=45$ & \\
\hline Lesion depth & $4.0[2.7-4.5]$ & $4.4[3.3-5.4]$ & 0.014 \\
\hline Surface area & $22.7[18.8-32.5]$ & $29.2[23.5-37.4]$ & 0.005 \\
\hline Lesion volume & $126.1[75.7-213.7]$ & $175.1[97.8-281.0]$ & 0.018 \\
\hline Parallel & $\mathrm{N}=45$ & $\mathrm{~N}=45$ & \\
\hline Lesion depth & $3.8[2.9-4.2]$ & $3.9[3.1-4.7]$ & 0.28 \\
\hline Surface area & $28.7[24.2-39.3]$ & $30.1[26.7-33.8]$ & 0.78 \\
\hline Lesion volume & $165.3[101.2-229.9]$ & $175.0[106.7-274.8]$ & 0.63 \\
\hline \multicolumn{4}{|l|}{$30 \mathrm{~W}$} \\
\hline Total & $\mathrm{N}=89$ & $\mathrm{~N}=82$ & \\
\hline Lesion depth & $4.7[3.3-5.2]$ & $5.0[3.8-6.1]$ & 0.019 \\
\hline Surface area & $26.9[22.1-37.3]$ & $30.9[26.7-35.7]$ & 0.23 \\
\hline Lesion volume & 204.8 [131.0-332.5] & $281.4[145.0-422.3]$ & 0.011 \\
\hline Perpendicular & $\mathrm{N}=44$ & $\mathrm{~N}=41$ & \\
\hline Lesion depth & $4.6[3.5-5.1]$ & $5.4[4.1-6.3]$ & 0.015 \\
\hline Surface area & $23.2[20.0-26.3]$ & $28.7[22.3-32.9]$ & 0.009 \\
\hline Lesion volume & $183.2[107.7-227.9]$ & $304.3[156.2-437.8]$ & 0.002 \\
\hline Parallel & $\mathrm{N}=45$ & $\mathrm{~N}=41$ & \\
\hline Lesion depth & $4.9[3.2-5.3]$ & $4.6[3.5-5.9]$ & 0.39 \\
\hline Surface area & $34.6[27.1-46.2]$ & $32.7[28.6-37.8]$ & 0.25 \\
\hline Lesion volume & 241.8 [143.0-353.9] & 252.5 [143.4-382.6] & 0.66 \\
\hline \multicolumn{4}{|l|}{ 40W } \\
\hline Total & $\mathrm{N}=79$ & $\mathrm{~N}=38$ & \\
\hline Lesion depth & $5.0[4.0-6.0]$ & $5.0[4.3-6.0]$ & 0.437 \\
\hline Surface area & $34.0[28.3-44.6]$ & $42.1[33.7-51.7]$ & $<0.001$ \\
\hline Lesion volume & 268.8 [163.8-392.0] & $270.2[175.2-423.0]$ & 0.346 \\
\hline Perpendicular & $\mathrm{N}=41$ & $\mathrm{~N}=24$ & \\
\hline Lesion depth & $4.8[4.0-5.9]$ & $5.1[4.5-6.1]$ & 0.06 \\
\hline Surface area & $28.7[21.2-33.0]$ & 45.1 [39.1-54.2] & $<0.001$ \\
\hline Lesion volume & 216.5 [127.7-288.8] & $310.0[177.2-430.5]$ & 0.009 \\
\hline Parallel & $\mathrm{N}=38$ & $\mathrm{~N}=14$ & \\
\hline Lesion depth & $5.2[4.1-6.2]$ & $5.0[4.0-6.0]$ & 0.426 \\
\hline Surface area & $40.6[35.0-51.7]$ & $38.5[31.3-46.3]$ & 0.217 \\
\hline Lesion volume & $337.8[202.9-461.9]$ & 236.7 [180.6-418.8] & 0.201 \\
\hline
\end{tabular}

Table 3. Temperature control and average power by catheter placement

\begin{tabular}{llll}
\hline & Perpendicular & Parallel & P-value \\
\hline FlexAbility $^{\mathrm{TM}}$ SE & & & \\
Temperature control & & & \\
20W & 0 & 0 & 1 \\
$30 \mathrm{~W}$ & $14(31.1 \%)$ & $4(8.9 \%)$ & 0.016 \\
$40 \mathrm{~W}$ & $30(66.7 \%)$ & $12(26.7 \%)$ & $<0.001$
\end{tabular}

Average power (W) 


\begin{tabular}{llll}
\hline & Perpendicular & Parallel & P-value \\
\hline 20W & $20[19-20]$ & $19[19-20]$ & 0.26 \\
30W & $25[23-28]$ & $28[26-29]$ & $<0.001$ \\
40W & $31[27-35]$ & $36[33-38]$ & $<0.001$ \\
TactiCath ${ }^{\text {TM }}$ SE & & & \\
Temperature control & & & \\
20W & 0 & 0 & 1 \\
30W & 0 & 0 & 1 \\
40W & $3(6.7 \%)$ & 0 & 0.24 \\
Average power (W) & & & \\
20W & $20[19-20]$ & $20[19-20]$ & 0.83 \\
30W & $29[29-30]$ & $29[29-30]$ & 1 \\
$40 \mathrm{~W}$ & $38[38-39]$ & $39[38-39]$ & 0.003 \\
\hline
\end{tabular}

Table 4. Predictors of steam-pop

\begin{tabular}{|c|c|c|c|c|c|}
\hline & $\begin{array}{l}\text { Steam-Pop (-) } \\
\mathrm{N}=468\end{array}$ & $\begin{array}{l}\text { Steam-Pop } \\
(+) \mathrm{N}=72\end{array}$ & Univariate & Multivariate & OR $(95 \%$ CI $)$ \\
\hline Catheters & $\begin{array}{l}\text { TactiCath }^{\mathrm{TM}}: \\
210(44.9 \%) \\
\text { FlexAbility } \\
\text { SE: } 258(55.1 \%)\end{array}$ & $\begin{array}{l}\text { TactiCath }^{\text {TM }}: 60 \\
(83.3 \%) \\
\text { Flexablity }^{\text {TM }}: 12 \\
(16.7 \%)\end{array}$ & $<0.001$ & $<0.001$ & $\begin{array}{l}0.07(0.03-0.16) \\
\text { for } \\
\text { FlexAbility } \\
\text { SE }\end{array}$ \\
\hline Power & $\begin{array}{l}20 \mathrm{~W}: 180 \\
(38.5 \%) 30 \mathrm{~W}: \\
171(36.5 \%) \\
40 \mathrm{~W}: 117 \\
(25.0 \%)\end{array}$ & $\begin{array}{l}20 \mathrm{~W}: 0(0 \%) \\
30 \mathrm{~W}: 9 \\
(12.5 \%) 40 \mathrm{~W}: \\
63(87.5 \%)\end{array}$ & $\begin{array}{l}\text { Power }>40 \mathrm{~W} \\
<0.001\end{array}$ & $\begin{array}{l}\text { Power }>40 \mathrm{~W} \\
<0.001\end{array}$ & $\begin{array}{l}53.0 \\
(20.8-135.0)\end{array}$ \\
\hline Contact force & $\begin{array}{l}\text { 5g: } 164 \\
\text { (35.0\%) 15g: } \\
154(32.9 \%) \\
25 \mathrm{~g}: 150 \\
(32.1 \%)\end{array}$ & $\begin{array}{l}\text { 5g: } 16(22.2 \%) \\
\text { 15g: } 26 \\
\text { (36.1\%) 25g: } \\
30(41.7 \%)\end{array}$ & $\begin{array}{l}\text { Weight }>25 \mathrm{~g} \\
0.11\end{array}$ & $\begin{array}{l}\text { Weight }>25 \mathrm{~g} \\
0.047\end{array}$ & $2.1(1.0-4.2)$ \\
\hline $\mathrm{RF}$ duration & $\begin{array}{l}\text { 10s: } 106 \\
(22.6 \%) 20 \mathrm{~s}: \\
102(21.8 \%) \\
30 \mathrm{~s}: 90 \\
(19.2 \%) 45 \mathrm{~s}: \\
86(18.4 \%) \\
60 \mathrm{~s}: 84 \\
(17.9 \%)\end{array}$ & $\begin{array}{l}\text { 10s: } 2(2.8 \%) \\
\text { 20s: } 6(8.3 \%) \\
\text { 30s: } 18 \\
(25.0 \%) 45 \mathrm{~s}: \\
22(30.6 \%) \\
60 \mathrm{~s}: 24 \\
(33.3 \%)\end{array}$ & $\begin{array}{l}\text { Duration }>30 \mathrm{~s} \\
<0.001\end{array}$ & $\begin{array}{l}\text { Duration }>30 \text { s } \\
<0.001\end{array}$ & $8.0(3.6-17.4)$ \\
\hline Parallel & $128(48.8 \%)$ & $42(57.5 \%)$ & 0.16 & 0.045 & $2.1(1.0-4.1)$ \\
\hline $\begin{array}{l}\text { Impedance } \\
\text { drop }\end{array}$ & $17.5[13.8-23.0]$ & $26.0[20.0-29.3]$ & $\begin{array}{l}\text { Imp drop }>20 \Omega \\
<0.001\end{array}$ & $\begin{array}{l}\text { Imp drop }>20 \Omega \\
0.005\end{array}$ & $3.0(1.4-6.4)$ \\
\hline
\end{tabular}

\section{Figure legends}

Figure 1.

(A) Catheter tips of both catheters. The Flexibility ${ }^{\mathrm{TM}} \mathrm{SE}$ ablation catheter has a laser-cut 4-mm flexible $8 \mathrm{Fr}$ tip, which is irrigated from the proximal to distal end through the laser-cut kerfs. The TactiCath ${ }^{\mathrm{TM}} \mathrm{SE}$ 
ablation catheter has a 3.5-mm 8 Fr tip and has 6 -hole irrigation holes on the side of the tip. Both catheters have a single thermocouple embedded within the tip for temperature monitoring, and the distance from the tip is $0.3 \mathrm{~mm}$ in the FlexAbility ${ }^{\mathrm{TM}} \mathrm{SE}$ catheter and $2.64 \mathrm{~mm}$ in the TactiCath ${ }^{\mathrm{TM}} \mathrm{SE}$ catheter.

(B) Scheme of catheter orientation. The catheter was placed perpendicularly or parallelly to the tissue.

(C) Scheme of the surface and cross-sectioned lesion. Surface area and lesion volume were calculated from the following formulae:

surface area $=\pi \times \mathrm{a} / 2 \times \mathrm{b} / 2$.

lesion volume $=(1 / 6) \times \pi \times\left(e^{2} \times d+c \times a^{2} / 2\right)$.

A.

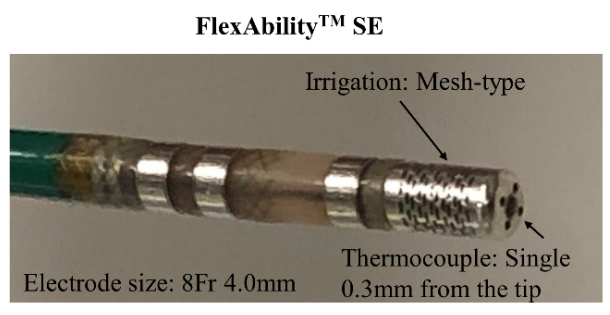

TactiCath $^{\text {TM }}$ Quartz

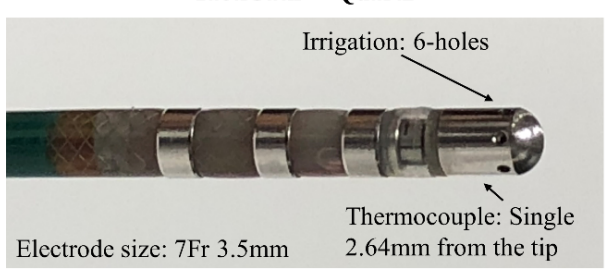

C.

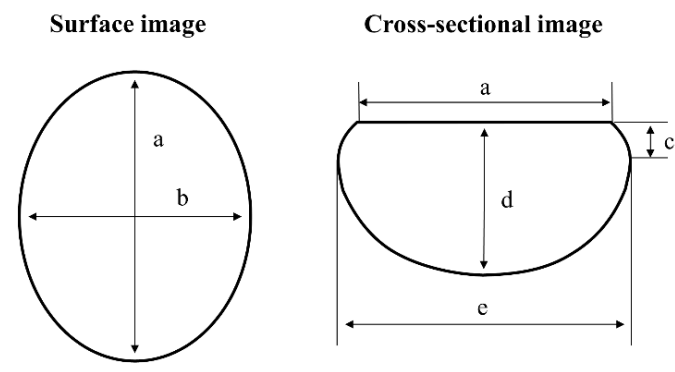

B.

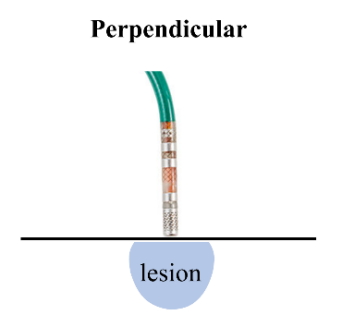

Parallel

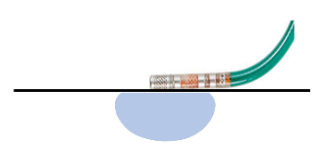

\section{Figure 2.}

Lesion volume for each power and catheter contact angle. In perpendicular lesions, lesion volume tended to be larger for the TactiCath ${ }^{\mathrm{TM}} \mathrm{SE}$ catheter than for the FlexAbility ${ }^{\mathrm{TM}}$ SE catheter. However for the parallel lesions, no significant differences were seen at any RF duration and power settings between catheters. 
A.

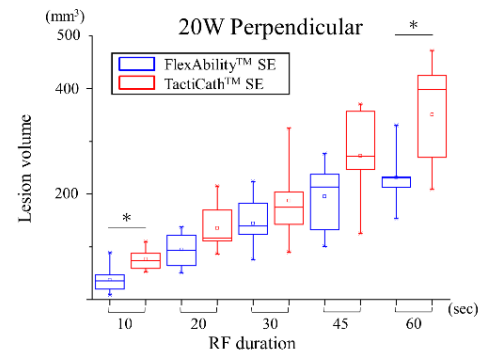

C.

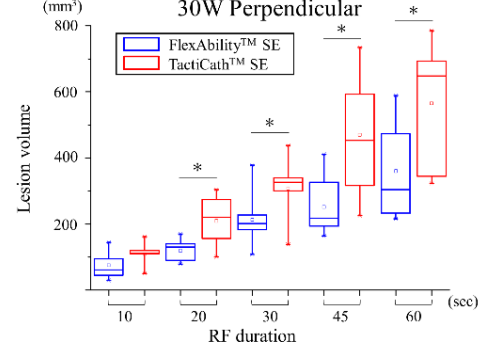

B.

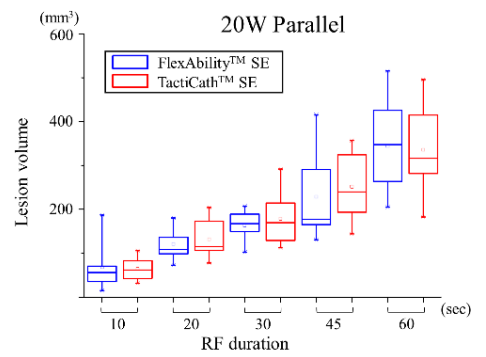

D.

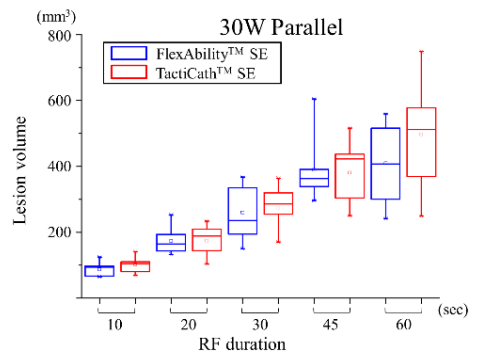

\section{Figure 3.}

(A) Sample image of cross-sectioned lesions. All lesions were created under the same settings of $30 \mathrm{~W}, 10 \mathrm{~g}$ and 30s duration. The left two lesions were created by perpendicular catheter placement, whereas the right two lesions were created by parallel catheter placement.

(B) Summary of the features of FlexAbility ${ }^{\mathrm{TM}} \mathrm{SE}$ and TactiCath $^{\mathrm{TM}} \mathrm{SE}$ catheter lesions. 
A.

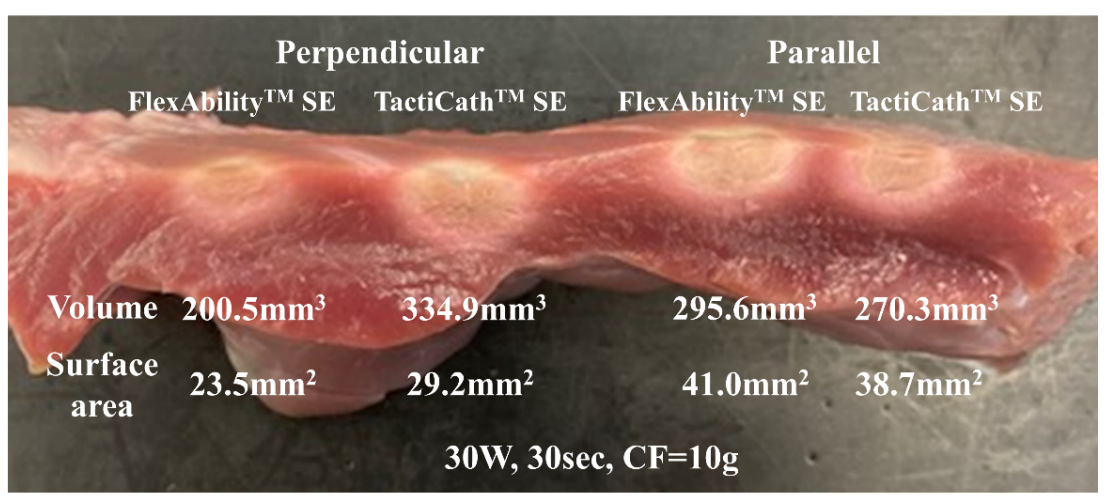

B.

\begin{tabular}{l|c} 
& FlexAbility $^{\mathrm{TM}}$ SE vs. TactiCath $^{\mathrm{TM}}$ SE \\
\hline Lesion size & $\leq$ \\
$\qquad \begin{array}{l}\text { Perpendicular } \\
\text { Parallel }\end{array}$ & $<$ \\
Temperature control & $>$ \\
& \\
Steam-pop & $<$ \\
Char formation &
\end{tabular}

\section{Figure 4.}

The images of irrigation flow of FlexAbility ${ }^{\mathrm{TM}} \mathrm{SE}$ using green dye.

(A) When the catheter is placed parallel to the tissue, irrigation flow spreads both from the proximal to distal end through laser-cut kerfs and from four holes on the distal end of the tip. 
B.

A.

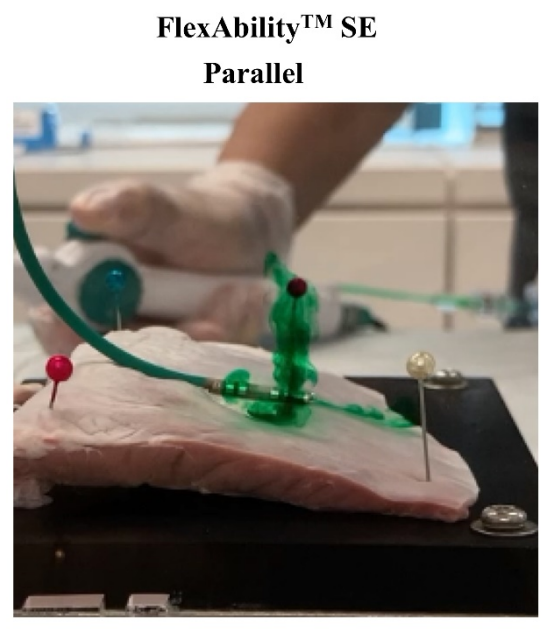

FlexAbility ${ }^{\mathrm{TM}} \mathrm{SE}$

Perpendicular

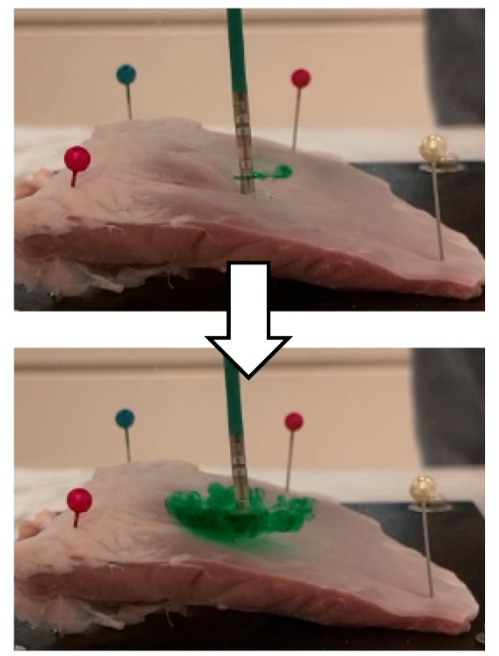

(B) When the catheter is placed perpendicular to the tissue, the distal holes were obstructed the by the tissue contact, resulted in irrigation flow spreading only from the proximal laser-cut kerfs, which may provide insufficient cooling.

\section{Supplementary Figure 1.}

Relationship between contact force measured from the TactiCath ${ }^{\mathrm{TM}} \mathrm{SE}$ catheter and weight on a scale. In the perpendicular angle lesions, weight on the scale had a very strong positive linear relationship to the CF on TactiCath ${ }^{\mathrm{TM}} \mathrm{SE}(\mathrm{R}=0.97)$, whereas in the parallel angle lesions, weight on the scale had a weaker yet still strong positive linear relationship $(\mathrm{R}=0.77)$.

A.

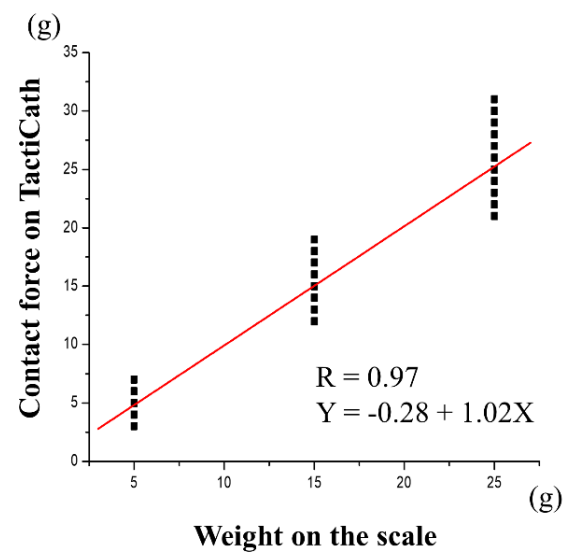

B.

(g)

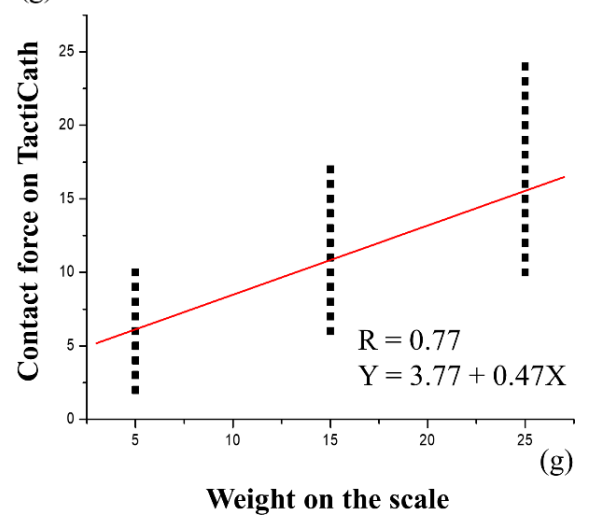

